\title{
Parameterized post-Newtonian orbital effects in extrasolar planets
}

\author{
Lin-Sen Li
}

Received: 2 March 2012 / Accepted: 4 April 2012 / Published online: 28 April 2012

(C) The Author(s) 2012. This article is published with open access at Springerlink.com

\begin{abstract}
Perturbative post-Newtonian variations of the standard osculating orbital elements are obtained by using the two-body equations of motion in the parameterized post-Newtonian theoretical framework. The results obtained are applied to the Einstein and Brans-Dicke theories. As a results, the semi-major axis and eccentricity exhibit periodic variation, but no secular changes. The longitude of periastron and mean longitude at epoch experience both secular and periodic shifts. The post-Newtonian effects are calculated and discussed for six extrasolar planets.
\end{abstract}

Keywords Parameterized post-Newtonian framework · Orbital effect in extrasolar planets

\section{Introduction}

At present, the post-Newtonian effect has been exhibited gradually in the wake of unceasing development in the post Newtonian celestial mechanics and due to that the accurate degree of astronomical instruments is heightened unceasingly. Hence some authors devoted to the research on the subject and scopes, such as Estabrook (1969), Nordtvedt (1970), Rubincam (1977), Brumberg (1972, 1985, 2010), Damour and Deruelle (1985), Soffel et al. (1987), Soffel (1989), Klioner and Kopejkin (1992), Calura et al. (1997), Brumberg et al. (1995), Brumberg and Brumberg (2001), Iorio (2005a, 2005b, 2007a, 2011a), Will (2008), Everitt et al. (2011), Kopeikin et al. (2011) and Iorio

L.-S. Li (ه)

Department of Physics, Northeast Normal University, Changchun, 130024, China

e-mail: dbsd_lls@yahoo.com.cn et al. (2011). In the post-Newtonian celestial mechanics there are some best methods One of the best methods is the method of parameterized post-Newtonian Formalism (PPN method) because the theories include the various different gravitational theories with different parameters, such as Einstein, Brans-Dicke and other theories. Hence some authors devoted to the research on this scope, such as, Misner et al. (1973), Nordtvedt (1976), Sarmiento (1982), Will (1981, 2006). Moreover, the Asymptotic method (Brumberg and Kopejkin 1989, 1990) and DSX method (Damour et al. 1991, 1992) are also desirable.

At present, some authors not only studied the postNewtonian effect on the motion of celestial objects in the solar system, but also in the extrasolar planetary system. It is interesting and significant for studying the post-Newtonian effects on the extrasolar planets because in the extrasolar planetary system the separation between planets and primary star is nearer mutually and planet mass is nearly Jupiter mass. Hence the post-Newtonian effect on the orbital elements of extraplanets is larger. In the recent years some authors studied the post-Newtonian effect or the relativistic effect in the extrasolar planets (Calura and Montanari 1999; Miralda-Escudé 2002; Wittenmyer et al. 2005; Iorio 2006, 2011b, 2011c; Adams and Laughlin 2006a, 2006b, 2006c; Heyl and Giadman 2007; Pál and Kocsis 2008; Jordán and Bakos 2008; Ragozzine and Wolf 2009). However, these authors used the method of the general relativity or the post-Newtonian approximation to study this problem. This paper used the parameterized post-Newtonian theories to study and calculate the parameterized post-Newtonian effect on the extrasolar planets with large eccentric orbit. 


\section{$2 R, S$ and $W$ components for the parameterized post-Newtonian perturbing acceleration in the two-body problem}

The relative acceleration of two-body with the post-Newtonian parameters is given by Will (1981)

$$
\begin{aligned}
\vec{a}_{P N}= & \frac{m \vec{X}}{r^{3}}\left[(2 \gamma+2 \beta) \frac{m}{r}-\gamma v^{2}+\left(2+\alpha_{1}-2 \zeta_{2}\right) \frac{\mu}{r}\right. \\
& -\frac{1}{2}\left(6+\alpha_{1}+\alpha_{2}+\alpha_{3}\right) \frac{\mu}{m} v^{2} \\
& \left.+\frac{3}{2}\left(\left(1+\alpha_{2}\right) \frac{\mu}{m}(\vec{v} \cdot \vec{n})^{2}\right)\right] \\
& +\frac{m(\vec{X} \cdot \vec{v}) \bar{v}}{r^{3}}\left[(2 \gamma+2)-\frac{\mu}{m}\left(2-\alpha_{1}+\alpha_{2}\right)\right] .
\end{aligned}
$$

Here

$$
\begin{aligned}
& m=m_{1}+m_{2}, \quad \mu=\frac{m_{1} m_{2}}{m}, \quad \tilde{N}=\frac{\vec{X}}{r}, \\
& \vec{X}=\vec{N} \cdot r=\vec{r}, \vec{r} \cdot \dot{\vec{t}}=r \cdot \dot{r}, \quad \vec{v}=\dot{\vec{r}}=\dot{r} \vec{N}+r \dot{f} \vec{\lambda}, \\
& v^{2}=\dot{r}^{2}+r^{2} \dot{f}^{2}, \\
& (\vec{v} \cdot \vec{N})^{2}=\left(\bar{v} \cdot \frac{\vec{X}}{r}\right)^{2}=\frac{(\vec{v} \cdot \vec{N} r)^{2}}{r^{2}}=\frac{\left(\dot{r}^{2} \cdot \vec{r}\right)^{2}}{r^{2}}=\dot{r}^{2}, \\
& \frac{m(\vec{X} \cdot \vec{v}) \vec{v}}{r^{3}}=\frac{m}{r^{3}}(\vec{N} r \cdot \vec{v}) \vec{v}=\frac{m}{r^{3}}(\vec{r} \cdot \dot{\vec{r}}) \dot{\vec{r}} \\
& \quad=\frac{m}{r^{3}}(r \cdot \dot{r})(\dot{r} \vec{N}+r \dot{f} \vec{\lambda})=r \dot{r} \vec{N}+r^{2} \dot{r} \dot{f} \vec{\lambda} .
\end{aligned}
$$

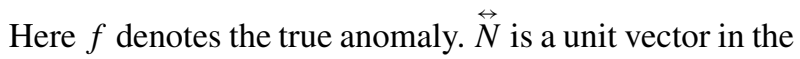
radial direction and $\vec{\lambda}$ are unit vectors in the orbital plane. $\overleftrightarrow{N}$ is directed along the radial direction, while $\vec{\lambda}$ is perpendicular to $\vec{N}$. In the equation $m$ denotes $G m$ and the right side should multipled by $c^{-2}$. $G$ is the gravitational constant and $\boldsymbol{c}$ is the speed of light. Equation (1) can be written as

$$
\begin{aligned}
\vec{a}_{p p n}= & \frac{m}{r^{3}}(r \dot{N})\left\langle(2 \gamma+2 \beta) \frac{m}{r}+\left(2+\alpha_{1}-2 \varsigma_{2}\right) \frac{\mu}{r}\right. \\
& -\left[\gamma+\frac{1}{2}\left(6+\alpha_{1}+\alpha_{2}+\alpha_{3}\right) \frac{\mu}{m}\right] \times\left(\dot{r}^{2}+r^{2} \dot{f}^{2}\right) \\
& \left.+\frac{3}{2}\left(1+\alpha_{2}\right) \frac{\mu}{m} \cdot \dot{r}^{2}\right\rangle \\
& +\frac{m}{r^{3}}\left[\left(r \dot{r}^{2} \dot{N}+r^{2} \dot{r} f \vec{\lambda}\right)\{(2 \gamma+2)\right. \\
& \left.\left.-\left(2-\alpha_{1}+\alpha_{2}\right) \frac{\mu}{m}\right\}\right] .
\end{aligned}
$$

Here $\rightarrow$ denotes vector.

We resolve the acceleration $\vec{a}$ into a radial component $R \overleftrightarrow{N}$, a component $S \vec{\lambda}$, normal to $R \overleftrightarrow{N}$ and a component $W$ normal to the orbital plane, i.e., $\vec{a}=R \overleftrightarrow{N}+S \vec{\lambda}+W(\overleftrightarrow{N} \times \vec{\lambda})$ $\overleftrightarrow{N} \times \vec{\lambda}=\overleftrightarrow{L}$ (the unit vector normal to the orbital plane).

On comparison with the expression (2), we get three scalar accelerative components $R, S$ and $W$

$$
\begin{aligned}
R= & \frac{m}{r^{2}}\left\langle(2 \gamma+2 \beta) \frac{m}{r}+\left(2+\alpha_{1}-2 \zeta_{2}\right) \frac{\mu}{r}\right. \\
& -\left[\gamma+\frac{1}{2}\left(6+\alpha_{1}+\alpha_{2}+\alpha_{3}\right) \frac{\mu}{m}\right] \\
& \left.\times\left(\dot{r}^{2}+r^{2} \dot{f}^{2}\right)+\frac{3}{2}\left(1+\alpha_{2}\right) \dot{r}^{2} \frac{\mu}{m}\right\rangle \\
& +\frac{m}{r^{2}} \dot{r}^{2}\left[\{2 \gamma+2)-\left(2-\alpha_{1}+\alpha_{2}\right) \frac{\mu}{m}\right], \\
S= & \frac{m}{r}\left[(2 \gamma+2) .-\left(2-\alpha_{1}+\alpha_{2}\right) \frac{\mu}{m}\right] \dot{r} \dot{f}, \\
W= & 0 .
\end{aligned}
$$

Substituting the following formulas of the problem of two body into the above formula (Smart 1953)

$\dot{f}=\frac{d f}{d t}=n a \sqrt[2]{1-e^{2}} / r^{2}, \quad \dot{r}=\frac{n a e \sin f}{\sqrt{1-e^{2}}}$,

$n^{2} a^{3}=m$.

We obtain

$$
\begin{aligned}
R= & \frac{m^{2}}{r^{2}}\left(\frac{2(\gamma+\beta)+\left(2+\alpha_{1}-2 \zeta_{2}\right) \frac{\mu}{m}}{r}\right. \\
& +\left[(\gamma+2)-\frac{1}{2}\left(7-\alpha_{1}+\alpha_{3}\right) \frac{\mu}{m}\right] \frac{e^{2} \sin ^{2} f}{p} \\
& \left.-\left[\gamma+\frac{1}{2}\left(6+\alpha_{1}+\alpha_{2}+\alpha_{3}\right) \frac{\mu}{m}\right] \frac{p}{r^{2}}\right), \\
S= & \frac{m^{2}}{r^{3}}\left[(2 \gamma+2)-\left(2-\alpha_{1}+\alpha_{2}\right) \frac{\mu}{m}\right] e \sin f, \\
W & =0
\end{aligned}
$$

where $p=a\left(1-e^{2}\right)$.

We can write simply the above formulas as the following formulas:

$$
\left\{\begin{array}{l}
r^{2} R=\left(\frac{K_{1}}{r}+K_{2} \frac{e^{2}}{p} \sin ^{2} f+K_{3} \frac{p}{r^{2}}\right), \\
r^{2} S=\frac{K_{4}}{r} e \sin f \\
W=0 .
\end{array}\right.
$$


Substituting $r=p /(1+e \cos f)$ into the right hand sides of the expression (2), we obtain

$$
\left\{\begin{aligned}
r^{2} R= & \frac{1}{p}\left\{\left[K_{1}+\frac{1}{2} e^{2} K_{2}+\left(1+\frac{1}{2} e^{2}\right) K_{3}\right]\right. \\
& \left.+\left(K_{1}+2 K_{3}\right) e \cos f+\frac{1}{2}\left(K_{3}-K_{2}\right) e^{2} \cos 2 f\right\} \\
r^{2} S= & \frac{1}{p}\left[K_{4}(1+e \cos f) e \sin f\right] \\
= & \frac{c^{2}}{p}\left(K_{4} e \sin f+\frac{1}{2} K_{4} e^{2} \sin 2 f\right) \\
W= & 0 .
\end{aligned}\right.
$$

In it, it is

$$
\left\{\begin{array}{l}
K_{1}=(2 \gamma+2 \beta) m^{2}+\left(2+\alpha_{1}-2 \zeta_{2}\right) m_{1} m_{2} \\
K_{2}=(\gamma+2) m^{2}-\frac{1}{2}\left(7-\alpha_{1}+\alpha_{3}\right) m_{1} m_{2} \\
K_{3}=-\gamma m^{2}-\frac{1}{2}\left(6+\alpha_{1}+\alpha_{2}+\alpha_{3}\right) m_{1} m_{2} \\
K_{4}=(2 \gamma+2) m^{2}-\left(2-\alpha_{1}+\alpha_{3}\right) m_{1} m_{2}
\end{array}\right.
$$

Based on the post-Newtonian parameters (Will 1981), in the general relativity the post-Newtonian parameters $\alpha_{1}=\alpha_{2}=$ $\alpha_{3}=0, \beta=1, \gamma=1, \zeta_{2}=0$. and in the Brans-Dicke gravitational theories $\alpha_{1}=\alpha_{2}=\alpha_{3}=0, \varsigma_{2}=0, \beta=1, \gamma=\frac{1+\omega}{2+\omega}$, $\omega$ is the dimensionless constant of the theory, $\omega=5$ (Estabrook 1969; Nordtvedt 1970).

\section{The post-Newtonian perturbing equations and the perturbing variables}

Substituting the perturbing accelerations $R, S, W$ for the formulas (3), into the following Gaussian equations (Brouwer and Clemence 1961)

$$
\left\{\begin{aligned}
& \frac{d a}{d t}= \frac{2}{n\left(1-e^{2}\right)^{1 / 2}}\left(\operatorname{Re} \sin f+S \frac{p}{r}\right), \\
& \frac{d e}{d t}= \frac{\left(1-e^{2}\right)^{1 / 2}}{n a}[R \sin f+S(\cos u+\cos f)], \\
& \frac{d I}{d t}= \frac{1}{n a\left(1-e^{2}\right)^{1 / 2}} W \frac{r}{a} \cos (\omega+f), \\
& \sin I \frac{d \Omega}{d t}=\frac{1}{n a\left(1-e^{2}\right)^{1 / 2}} W \frac{r}{a} \sin (\omega+f), \\
& \frac{d \tilde{\omega}}{d t}=\frac{\left(1-e^{2}\right)^{1 / 2}}{n a e}\left[-R \cos f+S\left(\frac{p}{r}+1\right) \sin f\right] \\
& \quad+2 \sin ^{2} \frac{1}{2} I \frac{d \Omega}{d t}, \\
& \frac{d \varepsilon}{d t}=-\frac{2 r}{n a^{2}} R+\frac{e^{2}}{1+\left(1-e^{2}\right)^{1 / 2}} \frac{d \omega}{d t} \\
&+2\left(1-e^{2}\right)^{1 / 2} \sin ^{2} \frac{1}{2} I \frac{d \Omega}{d t} .
\end{aligned}\right.
$$

Here $u$ is the eccentric anomaly. $\tilde{\omega}$ is the longitude of periastron and $\omega$ is the argument of periastron. $\varepsilon$ is the mean longitude at epoch.

We obtain the set of the post-Newtonian perturbing equations 
In the set of (6) we transform independent variable time $t$ into independent variable anomaly $f$ by using $d t=$ $r^{2} d f / n a \sqrt[2]{1-e^{2}}$ and $n^{2} a^{3}=m$, and then, integrating the equations

$\delta \sigma=\int_{\sigma_{0}}^{\sigma} d \sigma=\int_{f_{0}}^{f}\left[\frac{d \sigma}{d t} \frac{d t}{d f}\right] d f$.

In it $\sigma$ denotes arbitrary orbital elements from $a, e, \omega, i, \Omega$, and $\varepsilon_{0}$.

Substituting $\frac{d \sigma}{d t}$ for the set of (6) and $\frac{d t}{d f}=r^{2} / n a \times$ $\sqrt[2]{1-e^{2}}$ into the above definite integral expressions and integrating, one obtain the perturbation variables

$$
\begin{aligned}
& \delta \delta=-\frac{2}{m\left(1-e^{2}\right)^{2}}\left\{\left[K_{1}+\frac{3}{4} e^{2} K_{2}+\left(1+\frac{1}{4} e^{2}\right) K_{3}\right.\right. \\
& \left.+\left(1+\frac{1}{4} e^{2}\right) K_{4}\right] e\left(\cos f-\cos f_{0}\right) \\
& +\frac{1}{4}\left(K_{1}+2 K_{3}+2 K_{4}\right) e^{2}\left(\cos 2 f-\cos 2 f_{0}\right) \\
& \left.+\frac{1}{12}\left(K_{3}-K_{2}+K_{4}\right) e^{3}\left(\cos 3 f-\cos 3 f_{0}\right)\right\} \text {, } \\
& \delta e=-\frac{1}{m p}\left\{\left[K_{1}+\frac{3}{4} e^{2} K_{2}+\left(1+\frac{1}{4} e^{2}\right) K_{3}\right.\right. \\
& \left.+\frac{5}{4} e^{2} K_{4}\right]\left(\cos f-\cos f_{0}\right) \\
& +\frac{1}{4}\left(K_{1}+2 K_{3}+2 K_{4}\right) e\left(\cos 2 f-\cos 2 f_{0}\right) \\
& \left.+\frac{1}{12}\left(K_{3}-K_{2}+K_{4}\right) e^{2}\left(\cos 3 f-\cos 3 f_{0}\right)\right\} \text {, } \\
& \delta I=\delta \Omega=0 \\
& \delta \tilde{\omega}=\frac{1}{m p e}\left\{\left(K_{4}-\frac{1}{2} K_{1}-K_{3}\right) e\left(f-f_{0}\right)\right. \\
& +\left[\frac{1}{4} e^{2} K_{4}-\left(\frac{3}{4} e^{2}+1\right) K_{3}-\frac{1}{4} e^{2} K_{2}-K_{1}\right] \\
& \times\left(\sin f-\sin f_{0}\right) \\
& -\frac{1}{2}\left(\frac{1}{2} K_{1}+K_{3}+K_{4}\right) e\left(\sin 2 f-\sin 2 f_{0}\right) \\
& \left.-\frac{1}{12}\left(K_{4}+K_{3}-K_{2}\right) e^{2}\left(\sin 3 f-\sin 3 f_{0}\right)\right\}, \\
& \delta \varepsilon=\frac{1}{m p e}\left\{\left[\left(K_{4}-K_{3}-\frac{1}{2} K_{1}\right)\right.\right. \\
& \left.-\sqrt{1-e^{2}}\left(\frac{3}{2} K_{1}+2 K_{2}+K_{3}+K_{4}\right)\right] e\left(f-f_{0}\right) \\
& +2\left(1-e^{2}\right) e K_{2}\left(u-u_{0}\right)+\left(1-\sqrt{1-e^{2}}\right)\left[\frac{1}{4} e^{2} K_{4}\right.
\end{aligned}
$$

$$
\begin{aligned}
& \left.-\frac{3}{4}\left(e^{2}+1\right) K_{3}-\frac{1}{4} e^{2} K_{2}-K_{1}\right] \\
& -2 e \sqrt[2]{1-e^{2}}\left(K_{3}-K_{2}\right)\left(\sin f-\sin f_{0}\right) \\
& -\frac{1}{2}\left(1-\sqrt{1-e^{2}}\right)\left(\frac{1}{2} K_{1}+K_{3}+K_{4}\right) \\
& \times e\left(\sin 2 f-\sin 2 f_{0}\right) \\
& -\frac{1}{12}\left(1-\sqrt{1-e^{2}}\right)\left(K_{4}+K_{3}-K_{2}\right) e^{2} \\
& \left.\times\left(\sin 3 f-\sin 3 f_{0}\right)\right\}
\end{aligned}
$$

$\delta l=n\left(t-t_{0}\right)+\delta \varepsilon . u$ is the eccentric anomaly

Here $l$ denotes the mean longitude of periastron

The perturbative solutions (12) and (13) of Keplerian elements include over ten kinds of gravitational theories as shown in Table 5.1 in Will (1981). Hence the formals (12)(13) are important and worth-while. In this paper we only select two kind theories of general relativity and Brans-Dicke.

In the above last integral expression, we have used already the next integral expressions

$$
\begin{aligned}
& \int r d f=a \int \sqrt{1-e^{2}} d u \\
& \int \frac{e^{2} \sin ^{2} f}{p} r d f=\frac{\left(e^{2}-1\right)}{p} \int r d f+\int d f-e \int \cos f d f .
\end{aligned}
$$

Here $u$ is the eccentric anomaly.

\section{The secular variations of the orbital elements}

It is seen from the results of the integration (8) that there exist the secular terms for $\delta \omega$ and $\delta \varepsilon_{0}$

$$
\begin{aligned}
& \left(f-f_{0}\right)=n\left(t-t_{0}\right)+\text { Periodic terms } \\
& u-u_{0}=n\left(t-t_{0}\right)+\text { Periodic terms }
\end{aligned}
$$

Here $u$ denotes the eccentric anomaly and $u_{0}$ is the value of $u$ as $t=0$.

All other terms are the periodic terms for $\delta a, \delta e, \delta \tilde{\omega}$ and $\delta \varepsilon$. The coefficients of the periodic terms are the amplitudes of the periodic terms.

It is interesting for studying the secular terms. Hence we take the secular terms from the expressions (8) or integrating the definite integration (7) and taking the lower limit $f_{0}=0$ 
and upper limit $f=2 \pi$, the results of integration are that the periodic terms disappear and the secular terms appear per cycle by letting $m=G M / c^{2}$, we get

$$
\begin{aligned}
& \Delta a= \Delta e=\Delta I=\Delta \Omega=0 \\
& \Delta \tilde{\omega}=-\frac{2 \pi}{m p}\left(K_{4}-K_{3}-\frac{1}{2} K_{1}\right) \mathrm{rad} / \text { cycle } \\
& \Delta \varepsilon=-\frac{2 \pi}{m p}\left[K_{4}-K_{3}-\frac{1}{2} K_{1}+2\left(1-e^{2}\right) K_{2}\right. \\
&\left.-\sqrt{1-e^{2}}\left(\frac{3}{2} K_{1}+2 K_{2}+K_{3}+K_{4}\right)\right] \mathrm{rad} / \text { cycle } \\
& \Delta l=2 \pi+\Delta \varepsilon \\
& \Delta \tilde{\omega}=\Delta \omega+\Delta \Omega=\Delta \omega
\end{aligned}
$$

The time variation of periastron passage, $\tau$, can be derived from the following relation

$$
\begin{aligned}
& M_{0}=\varepsilon-\omega-\Omega, \quad M_{0}=-n \tau, \\
& -n \Delta \tau-\tau \Delta n=\Delta \varepsilon-\Delta \omega-\Delta \Omega, \\
& \Delta n=-\frac{3}{2}\left(\frac{n}{a}\right) \Delta a=0, \quad \Delta \Omega=0, \quad n=\frac{2 \pi}{P}, \\
& \Delta \tau=-\frac{1}{n}(\Delta \varepsilon-\Delta \omega)=-\frac{P}{2 \pi}(\Delta \varepsilon-\Delta \omega) .
\end{aligned}
$$

The secular rates per year are

$$
\left\{\begin{array}{l}
\dot{a}=\dot{e}=\dot{I}=\dot{\Omega}=0, \\
\dot{\tilde{\omega}}=\Delta \tilde{\omega} / P(\mathrm{rad} / \mathrm{yr}), \\
\dot{\varepsilon}=\Delta \varepsilon / P(\mathrm{rad} / \mathrm{yr}), \\
\dot{l}=2 \pi / P+\Delta \varepsilon_{0} / P(\mathrm{rad} / \mathrm{yr}), \\
\dot{\tau}=\Delta \tau / P(\mathrm{~s} / \mathrm{yr}) .
\end{array}\right.
$$

Here $P$ denotes the orbital period, in yr.

Substituting $K_{1}, K_{2}, K_{3}$ and $K_{4}$ for the expressions (9) into the expressions (14) and by replacing $G$ and $c^{2}$, then, we obtain the formulas for the secular variables and the variable rate in the general relativity

$$
\left\{\begin{aligned}
\Delta \tilde{\omega}_{G R}= & \frac{6 \pi G m}{c^{2}\left(1-e^{2}\right)}(\mathrm{rad} / \text { cycle }), \\
\Delta \varepsilon_{G R}= & \frac{6 \pi G m}{c^{2} a\left(1-e^{2}\right)}\left[1+2\left(1-e^{2}\right)-5 \sqrt{1-e^{2}}\right] \\
& +\frac{2 \pi G m}{c^{2} a\left(1-e^{2}\right)} \\
& \times\left[9 \sqrt{1-e^{2}}-7\left(1-e^{2}\right)\right](\mathrm{rad} / \text { cycle }), \\
\Delta \tau_{G R}= & -\frac{1}{n}\left(\Delta \varepsilon_{G R}-\Delta \varpi_{G R}\right) \\
= & -\frac{P}{2 \pi}\left(\Delta \varepsilon_{0 G r}-\varpi_{G r r}\right)(\text { s/cycle })
\end{aligned}\right.
$$

\begin{tabular}{|c|c|c|c|c|c|c|}
\hline Exoplanets & $\begin{array}{l}\dot{\tilde{\tilde{\omega}}}_{G R} \\
\left({ }^{\prime \prime} / \mathrm{yr}\right)\end{array}$ & $\begin{array}{l}\dot{\varepsilon}_{G R} \\
(" / \mathrm{yr})\end{array}$ & $\begin{array}{l}\dot{\omega}_{B-D} \\
(" / \mathrm{yr})\end{array}$ & $\begin{array}{l}\dot{\varepsilon}_{B-D} \\
(" / \mathrm{yr})\end{array}$ & $\begin{array}{l}\dot{\varepsilon}_{G R} \\
(\mathrm{~s} / \mathrm{yr})\end{array}$ & $\begin{array}{l}\dot{\tau}_{B-D} \\
(\mathrm{~s} / \mathrm{yr})\end{array}$ \\
\hline HD68988b & $35.61^{\prime \prime}$ & -45.39 & 31.18 & -42.66 & 33.84 & 31.16 \\
\hline HD16874b & 35.19 & -46.60 & 31.83 & -43.18 & 34.81 & 32.14 \\
\hline HD217107b & 26.86 & -35.22 & 24.29 & -33.01 & -29.21 & 27.02 \\
\hline HD88133b & 106.64 & -153.94 & 96.76 & -128.73 & 56.24 & 51.13 \\
\hline $\mathrm{XO}-3 \mathrm{~b}$ & 142.46 & -176.26 & 125.86 & -155.30 & -66.22 & -59.89 \\
\hline GJ-346b & 83.19 & -103.19 & 71.97 & -95.04 & -31.89 & -29.46 \\
\hline
\end{tabular}

Table 1 The secular variations per revolution for the orbital elements of six extrasolar planets

Note: " denotes are second and: Rev denotes Revolution (Cycle)

Table 2 The secular rates per year for the orbital elements of six extrasolar planets

\begin{tabular}{lllllll}
\hline Exoplanets & $\begin{array}{l}\Delta \tilde{\omega}_{G R} \\
(" / \operatorname{Rev})\end{array}$ & $\begin{array}{l}\Delta \varepsilon_{G R} \\
(" / \operatorname{Rev})\end{array}$ & $\begin{array}{l}\Delta \tilde{\omega}_{B-D} \\
\left({ }^{\prime \prime} / \operatorname{Rev}\right)\end{array}$ & $\begin{array}{l}\Delta \varepsilon_{B-D} \\
\left({ }^{\prime \prime} / \operatorname{Re}\right)\end{array}$ & $\begin{array}{l}\Delta \varepsilon_{G R} \\
(\mathrm{~s} / \mathrm{yr})\end{array}$ & $\begin{array}{l}\Delta \tau_{B-D} \\
(\mathrm{~s} / \mathrm{yr})\end{array}$ \\
\hline HD68988b & $0.61^{\prime \prime}$ & $-0.78^{\prime \prime}$ & $0.55^{\prime \prime}$ & $-0.73^{\prime \prime}$ & -0.58 & 0.54 \\
HD16874b & 0.62 & -0.81 & 0.56 & -0.76 & -0.61 & 0.56 \\
HD217107b & 0.52 & -0.68 & 0.47 & -0.64 & 0.57 & 0.53 \\
HD88133b & 0.99 & -1.32 & -0.90 & -1.20 & -0.52 & 0.48 \\
XO-3b & 1.24 & -1.51 & 1.10 & -1.36 & -0.57 & 0.52 \\
GJ-436b & 0.60 & -0.74 & 0.52 & -0.69 & -0.23 & 0.21 \\
\hline
\end{tabular}


$\left\{\begin{array}{l}\dot{\tilde{\omega}}_{G R}=\Delta \tilde{\tilde{\omega}}_{G R} / P(\mathrm{rad} / \mathrm{yr}), \\ \dot{\varepsilon}_{G R}=\Delta \varepsilon_{G R} / P(\mathrm{rad} / \mathrm{yr}), \\ \dot{\tau}_{G R}=\Delta \tau_{G R} / P(\mathrm{~s} / \mathrm{yr}) .\end{array}\right.$

In the Brans-Dicke theory

$$
\begin{aligned}
& \left\{\begin{aligned}
& \Delta \dot{\tilde{\omega}}_{B-D}= \frac{38}{7} \frac{\pi G m}{c^{2}\left(1-e^{2}\right)}(\mathrm{rad} / \text { cycle }) \\
& \Delta \varepsilon_{B-D}= \frac{38}{7} \frac{\pi G m}{c^{2} a\left(1-e^{2}\right)}\left[1+\frac{40}{19}\left(1-e^{2}\right)\right. \\
&\left.-\frac{99}{19} \sqrt{1-e^{2}}\right]+\frac{4 \pi G m}{c^{2} a\left(1-e^{2}\right)} \\
& \times\left[6 \sqrt{1-e^{2}}-5\left(1-e^{2}\right)\right](\mathrm{rad} / \mathrm{cycle}) \\
& \Delta \tau_{B-D}=-\frac{P}{2 \pi}(\Delta \varepsilon-\Delta \varpi)(\mathrm{s} / \mathrm{cycle}) \\
& \dot{\tilde{\omega}}_{B-D}=\frac{\Delta \tilde{\varpi}_{B-D}}{P}(\mathrm{rad} / \mathrm{yr}) \\
& \dot{\varepsilon}_{B-D}=\frac{\Delta \varepsilon_{B-D}}{P}(\mathrm{rad} / \mathrm{yr}) \\
& \dot{\tau}_{B-D}=\frac{\Delta \tau_{B-D}}{P}(\mathrm{~s} / \mathrm{yr})
\end{aligned}\right.
\end{aligned}
$$

\section{Numerical calculation for six exotrasolar planets}

In this paper we choose six exoplanets: HD16871b, HD68988b, HD217107b, HD88133b, XO-3b and GJ-436b as an example for the former exoplanets, their $P(d)$, $M^{*}\left(M_{\odot}\right)$ are retrieved from Bodenheimer et al. (2003) and $a(\mathrm{Au}), e$ and $m_{\mathrm{b}}\left(m_{\mathrm{J}}\right)$ are cited from www.mpia.de/homes/ Lyra/planet_naming.html.; for latter three exoplanets, their $P(d) a(\mathrm{Au}), M^{*}\left(M_{\odot}\right)$ and $e$ are retrieved from Jordán and Bakos (2008) and $m_{\mathrm{b}}\left(m_{\mathrm{J}}\right)$ is cited from http://www. exoplanet.eu/index.php. These data are listed in Table 5 of the Appendix.

Substituting those data into formulas (17)-(20), we obtain the numerical results for the secular variation of the orbital element of six exoplanets in Table 1 and Table 2.

Table 3 Comparison with other authors for three exoplanets

\begin{tabular}{llll}
\hline Exoplanets & This study & $\begin{array}{l}\text { Jordán and Bakos (2008) } \\
\dot{\tilde{\omega}}(\mathrm{deg} / \mathrm{cy})\end{array}$ \\
\cline { 2 - 3 } & $\dot{\tilde{\omega}}\left({ }^{\prime \prime} / \mathrm{cy}\right)$ & $\dot{\dot{\tilde{\omega}}(\mathrm{deg} / \mathrm{cy})}$ & $2^{\circ} .958$ \\
HD88133b & $10664^{\prime \prime}$ & $2^{\circ} .961$ & $2^{\circ} .886$ \\
XO-3b & 14246 & $3^{\circ} .959$ & $2^{\circ} .234$ \\
GJ-436b & 8319 & $2^{\circ} .311$ & \\
\hline
\end{tabular}

\section{Discussion}

6.1 Comparison with the results of other authors

The results of the numerical values of advance of periastron of HD88133b, XO-3b and GJ-436b in this paper as compared with that of three exoplanets in the other author's work (Jordán and Bakos 2008) are listed in Table 3.

We can seen from the above Table 3 that both results are nearly approximate in the relativistic effect, but there are some different. The calculated values of this paper are some larger than that of Jordán and Bakos (2008) This difference results in that this paper calculates $\dot{\omega}_{G R}$ by using the mass of two-body (parent star and exoplanet) and Jordán and Bakos only consider the mass of the parent star and neglect the mass of exoplanet.

\subsection{Comparison with the planets in solar system}

Substituting the data of Mercury and Jupiter into the formulas (11) for $\Delta \tilde{\omega}_{E}$, we obtain the results for the comparison of the perihelion of Mercury and Jupiter per century with that of two exoplanets per century listed in Table 4.

We can see from Table 4 that the values of advance of the periastron of the exoplanets are largest than that of the planets in the solar system. Therefore, it is important and meaningful for studying the motion of the exoplanets.

\subsection{On the possibility of observing these effects}

Let us discuss the possibility of observing these effects. In the solar system the advance of perihelion of Mercury is $42.91^{\prime \prime}$ per century. We may see from Table 3 that in the extrasolar planetary system the maximal value of advance of XO-3b is $14246^{\prime \prime}$ per century which correspond to 332 time (fold) value of advance of perihelion of the Mercury. At present, some authors applied the method of TTV (transit timing variation) or the method of TDV (transit duration variation). i.e., the secular precession can be detected through the long-term change in $P_{\mathrm{obs}}$ or in $T_{\mathrm{D}}$ (TDV) to the observation of the extrasolar planetary system (Agol et al. 2005; Rabus et al. 2009; Gibson et al. 2009; Iorio 2011b). Therefore, the possibility that the non-Newtonian advances of the periastra of the extrasolar planets considered can be observed is certainly interesting and deserves further studies.

\subsection{Slouly orbiting planers}

The author emphasezes that when we consider slouly orbiting planers, we could look like secular term over relatively short observational time interval, i.e., the relatively short arcs or the short term effect are available and important. Hence the author takes the time interval per year in the Table 1 and Table 2. 
Table 4 The results of comparison with that of the Mercury and Jupiter

\begin{tabular}{llllll}
\hline Exoplanets & $\begin{array}{l}(\Delta \tilde{\omega})_{\max } \\
\left({ }^{\prime \prime} / \text { cy }\right)\end{array}$ & $\begin{array}{l}(\Delta \tilde{\omega})_{\min } \\
\left({ }^{\prime \prime} / \text { cy }\right)\end{array}$ & $\begin{array}{l}\text { Planets in solar } \\
\text { system }\end{array}$ & $\begin{array}{l}(\Delta \tilde{\omega})_{\max } \\
\left({ }^{\prime \prime} / \text { cy }\right)\end{array}$ & $\begin{array}{l}(\Delta \tilde{\omega})_{\text {small }} \\
\left({ }^{\prime \prime} / \text { cyt }\right)\end{array}$ \\
\hline $\begin{array}{l}\text { XO-3b } \\
\text { HD217107b }\end{array}$ & $14246^{\prime \prime}$ & & $\begin{array}{l}\text { Mercury } \\
\text { Jupiter }\end{array}$ & $42.91^{\prime \prime}$ & $0.06^{\prime \prime}$ \\
\hline
\end{tabular}

Table 5 Orbital and physical parameters of six extrasolar planetary systems used in the text

\begin{tabular}{|c|c|c|c|c|c|c|}
\hline Exoplanets & $P(d)$ & $a(\mathrm{Au})$ & $M^{*}\left(m_{\odot}\right)$ & $m_{\mathrm{b}}\left(m_{\mathrm{J}}\right)$ & $e$ & Ref. \\
\hline HD68988b & 6.276 & 0.071 & 1.11 & 1.90 & 0.140 & Bodenheimer et al. (2003) \\
\hline HD16874b & 6.403 & 0.065 & 1.09 & 0.23 & 0.081 & Bodenheimer et al. (2003) \\
\hline HD217107b & 7.125 & 0.073 & 0.98 & 1.33 & 0.132 & Bodenheimer et al. (2003) \\
\hline HD88133b & 3.416 & 0.047 & 1.20 & 0.22 & 0.133 & Jordán and Bakos (2008) \\
\hline $\mathrm{XO}-3 \mathrm{~b}$ & 3.192 & 0.048 & 1.41 & 11.79 & 0.260 & Jordán and Bakos (2008) \\
\hline GJ-436b & 2.644 & 0.028 & 0.41 & 0.0737 & 0.159 & Jordán and Bakos (2008) \\
\hline
\end{tabular}

$m_{\mathrm{b}}$ denotes exoplanet mass which is cited from http://www.mpia.de/homes/Lyra/planet_naming.html for the former three references, and the latter three references is cited from http://www.exoplanet.eu/index.php for $m_{\mathrm{b}}$

\subsection{Prospect for further investigation (the new try)}

At present, the fifth force, Yukawa-like interaction has been investigated in our solar system (Iorio 2007b; Haranas et al. 2011; Tsang 2012). It may be predicted that extrasolar planets may well be used also for constraining putative fifth force, Yukawa-like interaction in the further investigation.

\section{Conclusions}

In this paper we worked out parameterized post-Newtonian effect on the orbits of celestial objects. The semi-major axis and eccentricity exhibit periodic variation, but no secular variation. The longitude of periastron and mean longitude at epoch exhibit secular and periodic variation. The inclination and the longitude of ascending node are unaffected. Such effects on the orbits of the extrasolar planets may be observed possibly because their effects of advance of periastron are large as in the calculation of this paper. The results of this paper based on the parameterized post-Newtonian gravitational metric by the work of C.M. Will, amplified and extended his work.

Open Access This article is distributed under the terms of the Creative Commons Attribution License which permits any use, distribution, and reproduction in any medium, provided the original author(s) and the source are credited.

\section{Appendix}

See Table 5 .

\section{References}

Adams, F.C., Laughlin, G.: Effects of secular interactions in extrasolar planetary systems. Astrophys. J. 649(2), 992-1003 (2006a)

Adams, F.C., Laughlin, G.: Ralativistic effect in extrasolar planetary systems. Int. J. Mod. Phys. D 15, 2133-2140 (2006b)

Adams, F.C., Laughlin, G.: Long-term evolution of close planets including the effects of secular interaction. Astrophys. J. 649(2), 1004-1009 (2006c)

Agol, E., Steffen, J., Sari, R., Clarkson, W.: On detecting terrestrial planets with timing of giant planet transits. Mon. Not. R. Astron. Soc. 359, 567-579 (2005)

Bodenheimer, P., Laughlin, G., Lin, D.N.C.: On the radii of extrasolar giant planets. Astrophys. J. 592, 555-563 (2003)

Brouwer, D., Clemence, G.M.: Methods of Celestial Mechanics. Academic Press, New York and London (1961)

Brumberg, V.A.: Relativistic Celestial Mechanics. Nauka, Moscow (1972) (in Russian)

Brumberg, V.A.: Essential Relativistic Celestial Mechanics. Adam. Hilger, Bristol (1985)

Brumberg, V.A.: Relativistic celestial mechanics. Scholarpedia 5(8), 10669 (2010)

Brumberg, V.A., Kopejkin, S.M.: Relativistic reference system and motion of test bodies in the vicinity of the Earth. Nuovo Cimento B 103, 63-98 (1989)

Brumberg, V.A., Kopejkin, S.M.: Relativistic time scales in the solar system. Celest. Mech. Dyn. Astron. 48(1), 23-44 (1990)

Brumberg, V.A., Brumberg, E.V.: Elliptic anomaly in constructing long term and short-thermodynamical theories. Celest. Mech. Dyn. Astron. 80, 159-166 (2001)

Brumberg, E.V., et al.: Analytical linear perturbation theory for highly eccentric satellite orbits. Celest. Mech. Dyn. Astron. 61, 369-387 (1995)

Calura, M., Fortini, P., Montanari, E.: Post-Newtonian Lagrangian planetary equation. Phys. Rev. D, Part. Fields 56(8), 4782-4788 (1997)

Calura, M., Montanari, E.: Exact solution to the homogeneous Maxwell equations in the field of a gravitational wave in linearized theory. Class. Quantum Gravity 16(2), 643-652 (1999)

Damour, T., Deruelle: General relativistic celestial mechanics of binary systems 1: the post-Newtonian motion. Ann. Inst. Henri Poincaré Probab. Stat. 43, 107-302 (1985) 
Damour, T., Soffel, M., Xu, C.: General-relativistic celestial mechanics. 1. Method and definition of reference systems. Phys. Rev. D, Part. Fields 43(10), 3273-3307 (1991)

Damour, T., Soffel, M., Xu, C.: General-relativistic celestial mechanics II transtational equations of motion. Phys. Rev. D, Part. Fields 45(4), 1017-1044 (1992)

Estabrook, F.B.: Post-Newtonian n-body equations of the Brans-Dicke theory. Astrophys. J. 158 (1969)

Everitt, C.W.F., et al.: Gravity probe B: final result of a space experiment to text general relativity. Phys. Rev. Lett. 106, 221101 (2011)

Gibson, N.P., Pollacco, D., Simpson, E.K., et al.: A transit tining analysis of nine light curves of the exoplanet system TrES-3. Astrophys. J. 700(2), 1078-1085 (2009)

Haranas, I., Ragos, C., Mioc, V.: Yukawa-type potential effects in the anomalistic period of celestial bodies. Astrophys. Space Sci. 332, 107-113 (2011)

Heyl, J.S., Giadman, B.J.: Using long-term transit timing to detect terrestrial planets. Mon. Not. R. Astron. Soc. 377(4), 1511-1591 (2007)

Iorio, L.: On the possibility of measuring the post Newtonian gravitoelectric correction to the orbital period of a test body in a solar system scenario. Mon. Not. R. Astron. Soc. 359, 328-332 (2005a)

Iorio, L.: Is it possible to measure the Lense-Thirring effect on the orbit of planets in gravitational field of the sun. Astron. Astrophys. 431, 385-389 (2005b)

Iorio, L.: Are we far from testing general relativity with the transiting extrasolar planet HD 209458b "Osiris"? New Astron. 11, 490497 (2006)

Iorio, L.: The post-Newtonian mean anomaly advance as further postKeplerian parameter in pulsar binary systems. Astrophys. Space Sci. 312, 331-335 (2007a)

Iorio, L.: First preliminary text of the general relastivistic gravitomagnetic field of the sun and new constraints on a Yukawa-like fifth force from planetary data. Planeta ry Spase Sci. 55, 1290-1298 (2007b)

Iorio, L.: Long-term classical and relativistic affects on the radial velocities of the stars orbiting SqrA. Mon. Not. R. Astron. Soc. 411, 453-463 (2011a)

Iorio, L.: Classical and relativistic long-term time variation of some observables for transiting exoplanets. Mon. Not. R. Astron. Soc. 411, 167-183 (2011b)

Iorio, L.: Classical and relativistic node precessional effect in WASP33b and perspectives for detecting them. Astrophys. Space Sci. 331, 485-496 (2011c)

Iorio, L., et al.: Phenomenology of the Lense-Thirring effect in the solar system. Astrophys. Space Sci. 331, 351-395 (2011)

Jordán, A., Bakos, G.A.: Observability of the general relativistic precession of periastra in exoplanets. Astrophys. J. 685(1), 543-552 (2008)
Klioner, S.A., Kopejkin, S.M.: Microarcsecond astronometry in spacerelativistic effects and reduction of observation. Astron. J. 104(2), 897-914 (1992)

Kopeikin, S., Efroimsky, M., Kaplan, G.: Relativistic Celestial Mechanics of the Solar System. Wiley-VCH, Berlin (2011)

Miralda-Escudé, J.: Orbital perturbations of transiting planets: a possible method to measure stellar quadrupoles and to detect Earthmass planets. Astrophys. J. 564(2), 1019-1023 (2002)

Misner, C.W., Thorne, K.S., Wheeler, J.A.: Gravitation, Part IX, pp. 1066-1095. W.H. Freeman, San Francisco (1973)

Nordtvedt, K. Jr.: Post-Newtonian metric for a general class of scalartensor gravitational theories and observational consequences. Astrophys. J. 161, 1059-1067 (1970)

Nordtvedt, K. Jr.: Anisotropic parametrized post-Newtonian gravitational metric field. Phys. Rev. D, Part. Fields 14, 1311-1317 (1976)

Pál, A., Kocsis, B.: Periastron precession measurement in transiting extrasolar planetary systems at the level of general relativity. Mon. Not. R. Astron. Soc. 389(1), 191-198 (2008)

Ragozzine, D., Wolf, A.S.: Probing the interiors of very hot Jupiter's using transit light curves. Astrophys. J. 698(2), 1778-1794 (2009)

Rubincam, P.M.: General relativity and satellite orbit: the motion of a test particle in the Schwarzchild metric. Celest. Mech. Dyn. Astron. 15, 21-33 (1977)

Rabus, M., Deeg, H.J., Alonso, R., Belminte, J.A., Almenara, J.M.: Transit timing analysis of the exoplanets TrES-1 and TrES-2. Formalism for the solar system. Astron. Astrophys. 508(2), 10111020 (2009)

Sarmiento, A.F.: Parametrized post-post Newtonian $\left(\mathrm{PP}^{2} \mathrm{~N}\right)$ formalism for the solar system. Gen. Relativ. Gravit. 14, 793-805 (1982)

Smart, W.M.: Celestial Mechanics, p. 18, London, New York, Toronto (1953)

Soffel, M.H.: Relativity in Celestial Mechanics, Astrometry and Geodesy, Chap. 4. Springer, Heidelberg (1989)

Soffel, M.H., Ruder, H., Schneider, M.: The two-body problem in the (truncated) PPN theory. Celest. Mech. Dyn. Astron. 40(1), 77-85 (1987)

Tsang, L.M.: How can NASA's Lunar reconnaissance orbit projects verify the existence of the fifth force. New Astron. 17, 18-21 (2012)

Will, C.M.: Theory of Experiment in Gravitational Physics, Chaps. 4 6, pp. 86-165. Cambridge University Press, Cambridge (1981)

Will, C.M.: The confrontation between general relativity and experiment. Living Rev. Relativ. 9, 5-100 (2006)

Will, C.M.: Testing the general relativistic "No-Hair" theorems using the galactic center black hole Sagittarius A. Astrophys. J. Lett. 674, L25-L28 (2008)

Wittenmyer, R., Welsh, A., William, F., Orosz, J.A., et al.: System parameters of the transiting extrasolar planet HD 209458b. Astrophys. J. 632, 1157-1167 (2005) 